ESAIM: PROCEEDINGS AND SURVEYS, March 2015, Vol. 50, p. 113-132

Franck BOYER, Thierry GALLOUET, Raphaèle HERBIN and Florence HUBERT Editors

\title{
PERFECTLY MATCHED LAYERS IN NEGATIVE INDEX METAMATERIALS AND PLASMAS *
}

\author{
Éliane Bécache ${ }^{1}$, Patrick Joly ${ }^{1}$, Maryna Kachanovska ${ }^{1}$ and Valentin Vinoles ${ }^{1}$
}

\begin{abstract}
This work deals with the stability of Perfectly Matched Layers (PMLs). The first part is a survey of previous results about the classical PMLs in non-dispersive media (construction and necessary condition of stability). The second part concerns some extensions of these results. We give a new necessary criterion of stability valid for a large class of dispersive models and for more general PMLs than the classical ones. This criterion is applied to two dispersive models: negative index metamaterials and uniaxial anisotropic plasmas. In both cases, classical PMLs are unstable but the criterion allows us to design new stable PMLs. Numerical simulations illustrate our purpose.

Résumé. Ce travail porte sur la stabilité des Couches Absorbantes Parfaitement Adaptées (Perfectly Matched Layers, PMLs). La première partie est un récapitulatif de résultats antérieurs pour les milieux non dispersifs (construction et condition nécessaire de stabilité). La seconde partie concerne quelques extensions de ces résultats. Nous donnons un nouveau critère nécessaire de stabilité valable pour une grande classe de modèles dispersifs et pour des PMLs plus générales que celles classiques. Ce critère est appliqué à deux modèles dispersifs : les métamatériaux à indice négatif et les plasmas anistropiques uniaxiaux. Dans les deux cas, les PMLs classiques sont instables mais le critère nous permet de concevoir de nouvelles PMLs stables. Des simulations numériques illustrent notre propos.
\end{abstract}

\section{INTRODUCTION}

In the context of wave propagation in unbounded domains, the Perfectly Matched Layers (PMLs) are a very popular technique to construct artificial boundary conditions which absorb the outgoing waves. The idea is to surround the computational domain with an absorbing layer (the PML) which has the astonishing property of generating no reflection between the physical medium and the PML. Introduced by Bérenger [6] for the 3D Maxwell equations, the so-called classical PMLs have since shown their efficiency for many wave equations.

Unfortunately, there are several cases where such PMLs fail and lead to heavy instabilities. This is due to the presence of backward waves, i.e. waves whose phase and group velocities point in "opposite" directions with respect to the interface. In the case of non-dispersive media, a mathematical analysis has been performed and a necessary criterion of stability has been established: classical PMLs are stable only if there is no backward wave [3]. This result is obtained from an analysis of the dispersion relation and strongly relies on its homogeneity related to the absence of dispersion.

The case of dispersive media has been much less studied, at least from a mathematical point of view. The main difficulty is to deal with the dispersive nature of the models which makes the dispersion relation no more homogeneous.

* This work is partially supported by the ANR projects METAMATH (ANR-11-MONU-0016) and CHROME (ANR-12-CORD0013).

${ }^{1}$ POems team (UMR 7231 CNRS - Inria - Ensta ParisTech), Palaiseau, France

(C) EDP Sciences, SMAI 2015 
Our work focuses on two important dispersive models:

- Negative Index Metamaterials (NIMs), also called left-handed media or double negative metamaterials. These are artificial composite materials having extraordinary electromagnetic properties. In particular, they have negative permittivity and permeability at some frequencies due to microscopic resonating structures [20]. Since the 1990s, NIMs are the subject of intense researches due to their promising applications: superlens, cloaking, improved antenna, etc [10]. PMLs in such media have been already studied by the physics community $[9,11,14,19]$ but, to our knowledge, without complete mathematical justification.

- Cold Plasmas, also called non-thermal plasmas. In a nutshell, this model assumes the temperature of electrons and ions to be zero [7,21]. Such an approximation, according to Stix, provides "a remarkably accurate description of the common small-amplitude perturbations that are possible for hot plasmas" [21]. The applications are legion, one of the most important is controlling nuclear fusion. See for instance the ITER project ${ }^{1}$.

Since these models often show backward waves, we can expect that the classical PMLs will fail as they do for non-dispersive media. Our work consists in extending the results about the classical PMLs in non-dispersive media to such models.

This article is split into two parts:

(1) The first one consists in a survey of previous results about stability of classical PMLs in non-dispersive media. We first recall two ways to construct classical PMLs, using either a splitting of the unknowns or a change of variable. We then present a necessary criterion of stability for classical PMLs established in [3]. This criterion is interpreted geometrically and applied to some wave propagation models: isotropic and anisotropic acoustic equations, linearised Euler equations for aeroacoustic and elastodynamics.

(2) The second one is an overview of the extensions of these results to dispersive media and more general PMLs. We expose here the main ideas of some results which are proven in a forthcoming paper [5]. We first present two models we focus on: the Drude model (NIM) and the 2D uniaxial anisotropic plasma model (cold plasma). In particular, for both models, we demonstrate that backward waves exist and perform numerical simulations to illustrate the inherent instabilities of classical PMLs. Then we present a new necessary criterion of stability valid for a large class of dispersive models and for more general PMLs than the classical ones. This criterion helps us to design new stable PMLs for our two models. We finish by illustrating numerically the stability of these new PMLs.

\section{Classical PMLs for nON-Dispersive media: A SURVey of PRevious Results}

The goal of this section, which does not contain any new results, is to recall how to construct the classical PMLs for non-dispersive media and to present a necessary criterion of stability established previously in [3]. This criterion is interpreted geometrically and applied to some wave propagation models. We refer to [15] for an elementary introduction to the construction and the analysis of classical PMLs.

\subsection{Classical PMLs for non-dispersive media}

We start with a $2 \mathrm{D}$ hyperbolic system of unknown $\mathbf{u} \in \mathbb{R}^{m}$ of the form

$$
\partial_{t} \mathbf{u}+A_{x} \partial_{x} \mathbf{u}+A_{y} \partial_{y} \mathbf{u}=0,
$$

where $A_{x}$ and $A_{y}$ are $m \times m$ matrices with real entries. For simplicity, we assume here these matrices to be constant. The system is completed with an initial condition $\mathbf{u}(t=0)=\mathbf{u}_{0}$.

\footnotetext{
${ }^{1}$ http://www.iter.org
} 


\subsubsection{Classical PMLs by splitting of the unknown}

The formal construction, as Bérenger introduced it in [6], of the classical PMLs for (1) follows several steps which we recall here for a PML in the $x$ direction, i.e. when considering a physical domain in the half-space $x<0$ coupled with a PML in the half-space $x>0$ (see e.g. [15]).

- Splitting of the field. The field $\mathbf{u}$ is split into

$$
\mathbf{u}=\mathbf{u}^{x}+\mathbf{u}^{y}
$$

where $\left(\mathbf{u}^{x}, \mathbf{u}^{y}\right)$ satisfies the system

$$
\left\{\begin{array}{c}
\partial_{t} \mathbf{u}^{x}+A_{x} \partial_{x}\left(\mathbf{u}^{x}+\mathbf{u}^{y}\right)=0 \\
\partial_{t} \mathbf{u}^{y}+A_{y} \partial_{y}\left(\mathbf{u}^{x}+\mathbf{u}^{y}\right)=0
\end{array}\right.
$$

In this step, we do not add any damping, so that the split system (3) and the original system (1) are equivalent in the following sense: (i) if $\left(\mathbf{u}^{x}, \mathbf{u}^{y}\right)$ is solution of system (3) then obviously, by adding the two equations, $\mathbf{u}=\mathbf{u}^{x}+\mathbf{u}^{y}$ is solution of (1); (ii) reciprocally the solution $\mathbf{u}$ of system (1) can be decomposed as (2), where $\left(\mathbf{u}^{x}, \mathbf{u}^{y}\right)$ is solution of system (3) if one splits the initial data $\mathbf{u}_{0}$ in a compatible way. However, notice that the dimension of the unknown is doubled by this splitting.

- Adding a damping term. We add $\sigma \mathbf{u}^{x}$ with $\sigma=\sigma(x) \geq 0$ to the first equation of (3), the one which involves only the $x$ derivatives, which leads to

$$
\left\{\begin{array}{ccc}
\partial_{t} \mathbf{u}^{x}+\sigma \mathbf{u}^{x}+A_{x} \partial_{x}\left(\mathbf{u}^{x}+\mathbf{u}^{y}\right) & = & 0 \\
\partial_{t} \mathbf{u}^{y}+A_{y} \partial_{y}\left(\mathbf{u}^{x}+\mathbf{u}^{y}\right) & = & 0 .
\end{array}\right.
$$

In practice, one will choose of course $\sigma(x)=0$ for $x<0$ and $\sigma(x)>0$ for $x>0$. Moreover, we shall not apply the decomposition (2) in the physical half-space $x<0$ which means that one doubles the dimension of the unknown only in the PML region. The two physical and non physical media are coupled at the artificial interface $x=0$ by the transmission condition

$$
\mathbf{u}=\mathbf{u}^{x}+\mathbf{u}^{y} \quad \text { at } \quad x=0 .
$$

In the system (4), the two space directions $x$ and $y$ are not treated in the same way. In some sense, one applies an anisotropic absorption.

\subsubsection{PMLs seen as changes of variable}

Another way to obtain a system equivalent to (4) is to use a change of variable approach [8]. We start from the system (1) that we rewrite in the frequency domain (formally, we apply a time Fourier transform). We obtain the generalised Helmholtz equation

$$
i \omega \widehat{\mathbf{u}}+A_{x} \partial_{x} \widehat{\mathbf{u}}+A_{y} \partial_{y} \widehat{\mathbf{u}}=0
$$

Assume that $\widehat{\mathbf{u}}$ can be analytically extended to $x \in \mathbb{C}$. This function still satisfies $(6)$ for $\tilde{x} \in \mathbb{C}$ :

$$
i \omega \widehat{\mathbf{u}}(\tilde{x}, y)+A_{x} \partial_{x} \widehat{\mathbf{u}}(\tilde{x}, y)+A_{y} \partial_{y} \widehat{\mathbf{u}}(\tilde{x}, y)=0
$$

Constructing PMLs consists in considering the solution for $x>0$ on another complex path than the real half line, defined by a change of variable $\tilde{x}(x) \in \mathbb{C}(x \in \mathbb{R})$. The function $\mathbf{v}$ defined by

$$
\widehat{\mathbf{v}}(x, y)=\widehat{\mathbf{u}}(\tilde{x}(x), y), \quad x \in \mathbb{R},
$$


coincides with $\mathbf{u}$ in the left half plane $x<0$ as soon as

$$
\tilde{x}(x)=x \quad \text { for } \quad x<0 .
$$

This property ensures the perfect matching between $\mathbf{u}$ and $\mathbf{v}$. The function $\mathbf{v}$ satisfies

$$
i \omega \widehat{\mathbf{v}}(x, y)+A_{x} \alpha(x) \partial_{x} \widehat{\mathbf{v}}(x, y)+A_{y} \partial_{y} \widehat{\mathbf{v}}(x, y)=0,
$$

where we have introduced

$$
\alpha(x)=\left(\frac{\partial \tilde{x}(x)}{\partial x}\right)^{-1}
$$

which corresponds to the change of variable

$$
\tilde{x}(x)=\int_{0}^{x} \frac{1}{\alpha(\xi)} \mathrm{d} \xi
$$

Then (10) can be rewritten (after division by $i \omega$ ) as a splitting

$$
\widehat{\mathbf{v}}=\left(-\frac{\alpha(x)}{i \omega} A_{x} \partial_{x} \widehat{\mathbf{v}}\right)+\left(-\frac{1}{i \omega} A_{y} \partial_{y} \widehat{\mathbf{v}}\right):=\widehat{\mathbf{v}^{x}}+\widehat{\mathbf{v}^{y}}
$$

By construction, these two split functions satisfy

$$
\frac{i \omega}{\alpha} \widehat{\mathbf{v}^{x}}+A_{x} \partial_{x} \widehat{\mathbf{v}^{x}}=0 \quad \text { and } \quad i \omega \widehat{\mathbf{v}^{y}}+A_{y} \partial_{y} \widehat{\mathbf{v}^{y}}=0,
$$

which coincides, after going back in time, with (4) for the particular change of variable (11) with

$$
\alpha(x)=\left(1+\frac{\sigma(x)}{i \omega}\right)^{-1} .
$$

These two approaches, splitting the unknown and applying a change of variable, are thus equivalent.

\subsection{A necessary criterion of stability}

Well-posedness and stability of PMLs (at a continuous level) are two main mathematical questions that have been addressed for non-dispersive models in several papers (see e.g. [1-4], and [15] for a recap). For systems with constant coefficients, these two notions are both related to the solutions of the dispersion relation. We thus assume here $\sigma$ to be constant.

\subsubsection{Plane wave analysis}

One can show (e.g. [16]) that the well-posedness and stability amounts to the study of particular solutions which are harmonic plane waves

$$
\mathbf{u}=\mathbf{U} e^{i\left(\omega t-k_{x} x-k_{y} y\right)},
$$

where $\mathbf{U} \in \mathbb{R}^{m}$ is the amplitude vector, $\omega \in \mathbb{C}$ the angular frequency and $\mathbf{k}=\left(k_{x}, k_{y}\right) \in \mathbb{R}^{2}$ the wave vector. The study of harmonic plane waves in the original system (1) leads to the so-called dispersion relation

$$
F\left(\omega, k_{x}, k_{y}\right)=0 \quad \text { where } \quad F\left(\omega, k_{x}, k_{y}\right):=\operatorname{det}\left(i \omega I_{m}-i k_{x} A_{x}-i k_{y} A_{y}\right) .
$$

This is a polynomial equation of order $m$ in $\omega$. The $m$ solutions $\omega_{j}(\mathbf{k}), j=1, \ldots, m$, are called the modes. We shall assume in the following that they are real. For a given mode $\omega(\mathbf{k})$, we define its phase velocity as

$$
\mathbf{v}_{\mathbf{p}}(\omega(\mathbf{k}))=\frac{\omega(\mathbf{k})}{|\mathbf{k}|} \frac{\mathbf{k}}{|\mathbf{k}|}
$$


and its group velocity as

$$
\mathbf{v}_{\mathbf{g}}(\omega(\mathbf{k}))=\nabla_{\mathbf{k}} \omega(\mathbf{k})
$$

For isotropic non-dispersive models, the phase velocity and the group velocity are equal, but in general this is not necessarily true. Thus it is useful to introduce the following notions.

Definition 1.1. For a given direction $\mathbf{n}$, a mode $\omega$ is forward (resp. backward) in the $\mathbf{n}$ direction if

$$
\left(\mathbf{v}_{\mathbf{p}}(\omega) \cdot \mathbf{n}\right)\left(\mathbf{v}_{\mathbf{g}}(\omega) \cdot \mathbf{n}\right)>0 \quad\left(\operatorname{resp} . \quad\left(\mathbf{v}_{\mathbf{p}}(\omega) \cdot \mathbf{n}\right)\left(\mathbf{v}_{\mathbf{g}}(\omega) \cdot \mathbf{n}\right)<0\right)
$$

In particular, given a PML in the $x$ direction, a mode is forward (resp. backward) in the direction of the PML if it is forward (resp. backward) in the $\mathbf{e}_{x}$ direction.

\subsubsection{A necessary stability criterion}

First, we recall the notions of well-posedness and stability for the system (4).

Definition 1.2. The Cauchy problem (4) is weakly (resp. strongly) well-posed for any initial data $\left(\mathbf{u}_{0}^{x}, \mathbf{u}_{0}^{y}\right)=$ $\left(\mathbf{u}^{x}, \mathbf{u}^{y}\right)(t=0)$ given in the Sobolev space $H^{s}\left(\mathbb{R}^{2}\right), s>0$ (resp. $\left.s=0\right)$ if it admits a unique solution $\left(\mathbf{u}^{x}, \mathbf{u}^{y}\right)$ such that

$$
\left\|\left(\mathbf{u}^{x}, \mathbf{u}^{y}\right)(t)\right\|_{L^{2}\left(\mathbb{R}^{2}\right)} \leq C e^{\alpha t}\left\|\left(\mathbf{u}_{0}^{x}, \mathbf{u}_{0}^{y}\right)\right\|_{H^{s}\left(\mathbb{R}^{2}\right)}, \quad \alpha \in \mathbb{R} .
$$

It is weakly (resp. strongly) stable if it is weakly (or strongly) well-posed and we have an estimate

$$
\left\|\left(\mathbf{u}^{x}, \mathbf{u}^{y}\right)(t)\right\|_{L^{2}\left(\mathbb{R}^{2}\right)} \leq C(1+t)^{s}\left\|\left(\mathbf{u}_{0}^{x}, \mathbf{u}_{0}^{y}\right)\right\|_{H^{s}\left(\mathbb{R}^{2}\right)}
$$

The change of variable (11) with the particular choice (12), which corresponds to classical PMLs, transforms the dispersion relation (14) into

$$
F\left(\omega,\left(1+\frac{\sigma}{i \omega}\right)^{-1} k_{x}, k_{y}\right)=0
$$

It can be shown [16] that the (weak) well-posedness of a system of order $M$ which corresponds to (20) for a given $\sigma$ is ensured if all the modes $\omega_{j}(\mathbf{k}, \sigma)$ of $(20)$ are bounded from below:

$$
\exists C>-\infty, \forall \mathbf{k} \in \mathbb{R}^{2}, \forall j \in\{1, \ldots, M\}, \quad \operatorname{Im} \omega_{j}(\mathbf{k}, \sigma) \geq C,
$$

and the (weak) stability holds if and only if

$$
\forall \mathbf{k} \in \mathbb{R}^{2}, \forall j \in\{1, \ldots, M\}, \quad \operatorname{Im} \omega_{j}(\mathbf{k}, \sigma) \geq 0
$$

For non-dispersive models, the function $F$ in (14) is homogeneous with respect to $\omega, k_{x}$ and $k_{y}$. This property was heavily used in [3] to establish the so-called high-frequency necessary stability condition: analysing the sign of the imaginary part of the solutions $\omega(\mathbf{k}, \sigma)$ for small values of the damping $\sigma$ amounts in this case to analysing it for large frequencies $|\mathbf{k}|$.

Theorem 1.3 (high-frequency necessary stability condition). For the non-dispersive model (1), the necessary stability condition for the PML system in the $x$ direction (4) is that all the modes $\omega(\mathbf{k})$ solving the original dispersion relation (14) are forward in the $x$ direction, i.e.

$$
\forall \mathbf{k} \in \mathbb{R}^{2}, \quad\left(\mathbf{v}_{\mathbf{p}}(\omega(\mathbf{k})) \cdot \mathbf{e}_{x}\right)\left(\mathbf{v}_{\mathbf{g}}(\omega(\mathbf{k})) \cdot \mathbf{e}_{x}\right) \geq 0
$$

We call a system high-frequency stable if the condition (23) is satisfied. 


\subsubsection{Geometrical interpretation}

The criterion (23) can be interpreted geometrically. Since the function $F$ in (14) is homogeneous with respect to $\omega, k_{x}$ and $k_{y}$, one can plot the so-called slowness diagram which is the set of points $\mathbf{s}=\mathbf{k} / \omega$, the slowness vectors, such that

$$
F\left(1, \frac{\mathbf{k}}{\omega}\right)=F(1, \mathbf{s})=0 .
$$

Notice that the slowness vector $\mathbf{s}$ is collinear to the phase velocity $\mathbf{v}_{\mathbf{p}}(\omega)$ and points in the same direction. Moreover, it is easy to see that the group velocity is orthogonal to the slowness curves (24). Consequently, the condition (23), which can be written as

$$
\forall \mathbf{k} \in \mathbb{R}^{2}, \quad\left(\mathbf{s} \cdot \mathbf{e}_{x}\right)\left(\mathbf{v}_{\mathbf{g}}\left(\omega(\mathbf{k}) \cdot \mathbf{e}_{x}\right) \geq 0,\right.
$$

expresses the fact that, along the slowness curves, the slowness vector and the group velocity are oriented in the same way with respect to the $x$ direction. This gives a simple tool to check the necessary condition (23) (cf. Figure 1).
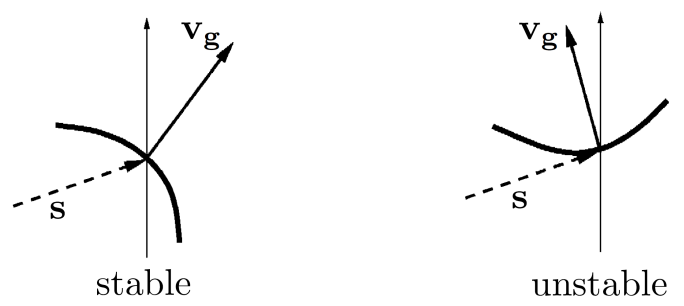

Figure 1. Left: the slowness vector $\mathbf{s}$ and the group velocity $\mathbf{v}_{\mathbf{g}}$ are oriented in the same way with respect to the $x$ direction. Right: $\mathbf{s}$ and $\mathbf{v}_{\mathbf{g}}$ are not oriented in the same way with respect to the $x$ direction.

\subsubsection{Application to some non-dispersive wave models}

- The isotropic acoustic equations. This model can be described by the first-order velocity pressure system

$$
\left\{\begin{aligned}
\partial_{t} p & =\nabla \cdot \mathbf{u} \\
\partial_{t} \mathbf{u} & =\nabla p
\end{aligned}\right.
$$

where $p$ is the acoustic pressure and $\mathbf{u}=\left(u_{x}, u_{y}\right)$ the flow velocity. For this model, the main slowness curve is simply a circle. Obviously, the geometrical criterion is satisfied (cf. Figure 2). Therefore the PMLs are high-frequency stable. A deeper analysis of PMLs in this context shows that they are actually stable (not only for high frequencies), see [4].

- The anisotropic acoustic equations. This model is the same as (25) but with an additional tensor:

$$
\left\{\begin{aligned}
\partial_{t} p & =\nabla \cdot \mathbf{u} \\
\partial_{t} \mathbf{u} & =A \nabla p
\end{aligned}\right.
$$

where $A$ is a $2 \times 2$ symmetric positive definite matrix

$$
A=\left[\begin{array}{ll}
a & b \\
b & c
\end{array}\right], \quad a>0, c>0, a c-b^{2}>0 .
$$

The main slowness curve of this model is an ellipse. Therefore, as soon as the axes are not parallel to the $x$ and $y$ axes, i.e. as soon as $b \neq 0$, the geometrically criterion is not satisfied (cf Figure 3). We refer to [12] for a construction of stable PMLs in this context. 


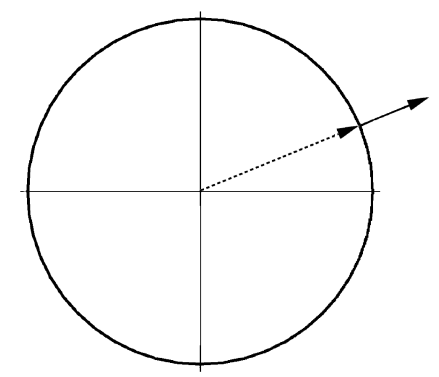

Figure 2. The slowness curve for the isotropic wave equation. The geometrical criterion is satisfied on every point of the curve.

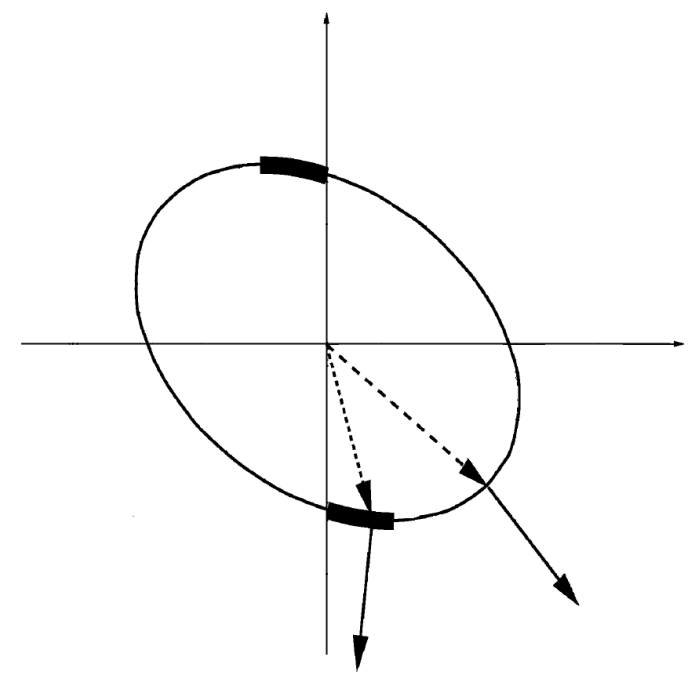

Figure 3. The slowness curve for the anisotropic wave equation. The geometrical criterion is not satisfied on the bold parts of the curve.

- The linearised Euler equations (aeroacoustic). This model describes an acoustic wave in an uniform subsonic flow (here parallel to the $x$ direction for simplicity) with the Mach number $0 \leq M<1$. This model is given by the following equation ( $p$ is the pressure and $\left(u_{x}, u_{y}\right)$ the velocity flow)

$$
\left\{\begin{array}{ccc}
\partial_{t} p+M \partial_{x} p & = & \partial_{x} u_{x}+\partial_{y} u_{y} \\
\partial_{t} u_{x}+M \partial_{x} u_{x} & = & \partial_{x} p \\
\partial_{t} u_{y}+M \partial_{x} u_{y} & = & \partial_{y} p .
\end{array}\right.
$$

In this case the main slowness curve is an ellipse with axes parallel to the coordinate axes but its center is $\left(M /\left(1-M^{2}\right), 0\right)$. Thus the geometrical criterion is not satisfied as soon as $M \neq 0$ (cf. Figure 4$)$. We refer to [13] for a deeper analysis and a construction of stable PMLs for this model.

- Elastodynamic equations. We deal with the velocity-stress formulation

$$
\left\{\begin{array}{l}
\partial_{t} \mathbf{u}=\nabla \cdot \sigma \\
\partial_{t} \sigma=C \varepsilon(\mathbf{u})
\end{array}\right.
$$

where $\mathbf{u}$ is the velocity field, $\sigma$ the stress tensor, $\varepsilon$ and $C$ the fourth order tensor of elastodynamic coefficients. In $2 \mathrm{D}$, the main slowness curves are composed of two branches, one corresponding to $\mathrm{P}$ 


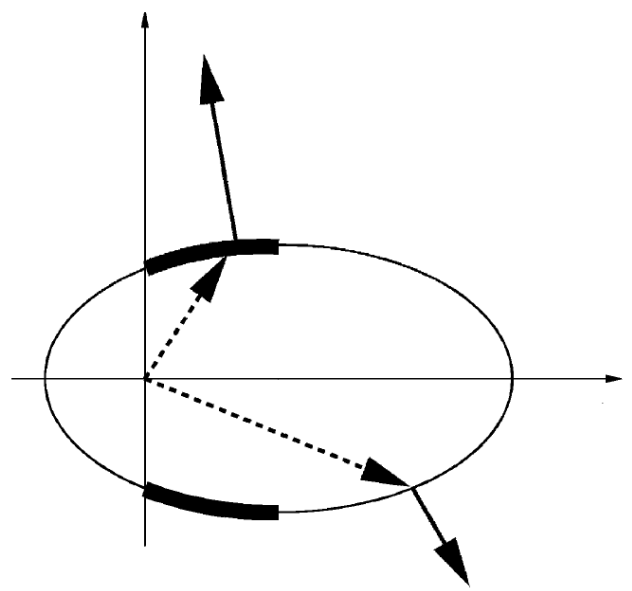

Figure 4. The slowness curve for the linearised Euler equation. The geometrical criterion is not satisfied on the bold parts of the curve.

waves and the second one to $\mathrm{S}$ waves. One can show that only $\mathrm{S}$ waves can give rise to high-frequency instabilities. The slowness curve corresponding to $\mathrm{S}$ waves can have very different shapes according to the values of the coefficients of $C$ (cf. Figure 5) so that the geometrical criterion could be satisfied or not. Thus the stability of the classical PMLs really depends on the properties of the medium. To our knowledge, the construction of stable PMLs for the general elastodynamics model which covers all the possible cases is still an open problem. We refer to [3] for a deeper analysis.
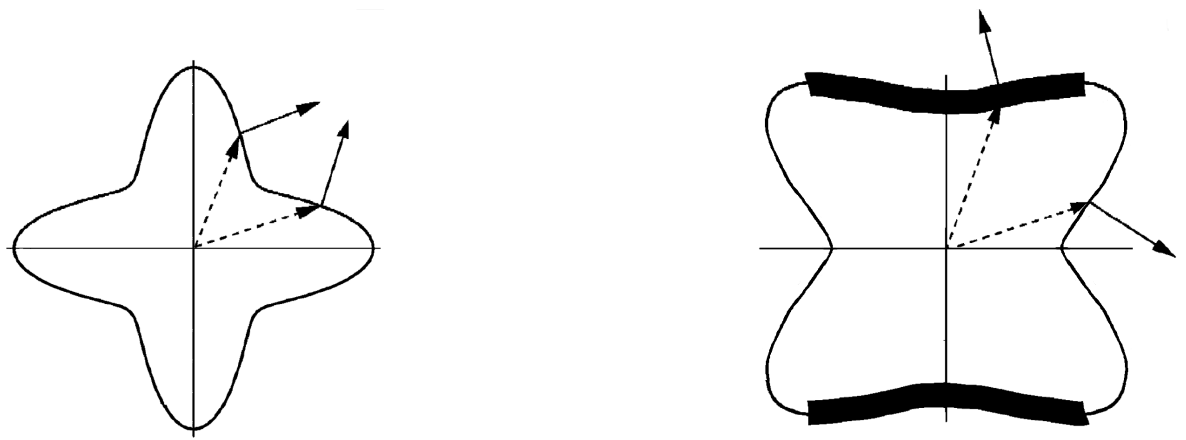

Figure 5. The slowness curves of $\mathrm{S}$ waves for two media. The geometrical criterion is satisfied for the left material but is not satisfied on the bold parts of the curve for the right one.

\section{Failure of Classical PMLs for some Dispersive Models With BaCKWARd WAVES}

Our work addresses the question of the construction of stable PMLs in dispersive media. This section presents the two dispersive models we will focus on. For both, a plane wave analysis shows that there are backward waves. By analogy with the previous non-dispersive case, we expect that classical PMLs are unstable for these two models. This is confirmed by the numerical simulations. 


\subsection{The case of negative index metamaterials: the Drude model.}

\subsubsection{The model}

The 2D Maxwell equations in the frequency domain (i.e. after a time Fourier transform) are

$$
\left\{\begin{array}{rlc}
i \omega \varepsilon(\omega) \widehat{E_{x}} & = & \partial_{y} \widehat{H} \\
i \omega \varepsilon(\omega) \widehat{E_{y}} & = & -\partial_{x} \widehat{H} \\
i \omega \mu(\omega) \hat{H} & = & \partial_{y} \widehat{E_{x}}-\partial_{x} \widehat{E_{y}}
\end{array}\right.
$$

where $\left(E_{x}, E_{y}\right)$ are the transverse electric fields, $H$ is the magnetic field and where $\varepsilon(\omega)$ is the permittivity and $\mu(\omega)$ the permeability which both depend on the frequency $\omega$ : the medium is dispersive. We allow both $\varepsilon(\omega)$ and $\mu(\omega)$ to take negative values. If there exists a frequency band where both $\varepsilon(\omega)$ and $\mu(\omega)$ are negative, the medium is called a Negative Index Metamaterial (NIM).

Remark 2.1. The notion of negative index comes from the fact that the index $n$ of a medium is defined by $n=(\varepsilon \mu)^{1 / 2}$ where the root is chosen using the causality principle. One can show that the simultaneous negativity of $\varepsilon$ and $\mu$ imposes that the root is actually negative [22].

From now on, we will focus on the Drude model

$$
\varepsilon(\omega)=\varepsilon_{0}\left(1-\frac{\omega_{e}^{2}}{\omega^{2}}\right), \quad \mu(\omega)=\mu_{0}\left(1-\frac{\omega_{m}^{2}}{\omega^{2}}\right),
$$

where $\varepsilon_{0}>0$ and $\mu_{0}>0$ are the permittivity and the permeability of the vacuum, and $\omega_{e}>0$ and $\omega_{m}>0$ are the electric and the magnetic plasma frequencies. The Drude model is the simplest model to describe negative index effects $[18,22]$ but it already contains one of the main difficulties to construct stable PMLs for NIMs, namely the presence of backward waves.

Depending on the values of $\omega$, the material shows different behaviours (cf. Figure 6). More precisely, there are three frequency bands:

- For $\omega>\max \left(\omega_{e}, \omega_{m}\right)$, one has $\varepsilon(\omega)>0$ and $\mu(\omega)>0$ : the material behaves as a standard dielectric medium. In particular $\varepsilon(\omega) \sim \varepsilon_{0}$ and $\mu(\omega) \sim \mu_{0}$ when $\omega \rightarrow+\infty$. Thus at high frequencies the medium is close to the vacuum.

- For $0<\omega<\min \left(\omega_{e}, \omega_{m}\right)$, one has $\varepsilon(\omega)<0$ and $\mu(\omega)<0$ : the material behaves as a NIM.

- If $\omega_{e} \neq \omega_{m}$, there is a band gap $\left(\min \left(\omega_{e}, \omega_{m}\right), \max \left(\omega_{e}, \omega_{m}\right)\right)$ of width $\left|\omega_{e}-\omega_{m}\right|$ where $\varepsilon$ and $\mu$ have opposite sign. This does not allow wave propagation.

\subsubsection{Plane waves analysis}

The dispersion relation of (26) with the Drude model (27) shows that, for a given wave vector $\mathbf{k}, \omega$ has to be a solution of

$$
|\mathbf{k}|^{2}=\frac{\omega^{2}}{c^{2}}\left(1-\frac{\omega_{e}^{2}}{\omega^{2}}\right)\left(1-\frac{\omega_{m}^{2}}{\omega^{2}}\right)
$$

where $c=1 / \sqrt{\varepsilon_{0} \mu_{0}}$ is the speed of light. The following proposition summarises how the modes behave for the Drude model.

Proposition 2.2. For all wave vectors $\mathbf{k} \in \mathbb{R}^{2}$, the equation (28) has 4 real distinct solutions $\pm \omega_{j}(\mathbf{k}), j=1,2$, given by

$$
\left\{\begin{array}{l}
\omega_{1}(\mathbf{k})=\frac{\sqrt{2}}{2} \sqrt{\omega_{e}^{2}+\omega_{m}^{2}+c^{2}|\mathbf{k}|^{2}+\sqrt{\Delta_{|\mathbf{k}|}}}, \\
\omega_{2}(\mathbf{k})=\frac{\sqrt{2}}{2} \sqrt{\omega_{e}^{2}+\omega_{m}^{2}+c^{2}|\mathbf{k}|^{2}-\sqrt{\Delta_{|\mathbf{k}|}}},
\end{array}\right.
$$

where $\Delta_{|\mathbf{k}|}=\left(\omega_{e}^{2}-\omega_{m}^{2}+c^{2}|\mathbf{k}|^{2}\right)^{2}+4 \omega_{m}^{2} c^{2}|\mathbf{k}|^{2}$. Moreover, one can show that the modes $\pm \omega_{1}(\mathbf{k})$ are forward in all the directions and the modes $\pm \omega_{2}(\mathbf{k})$ are backward in all the directions. 


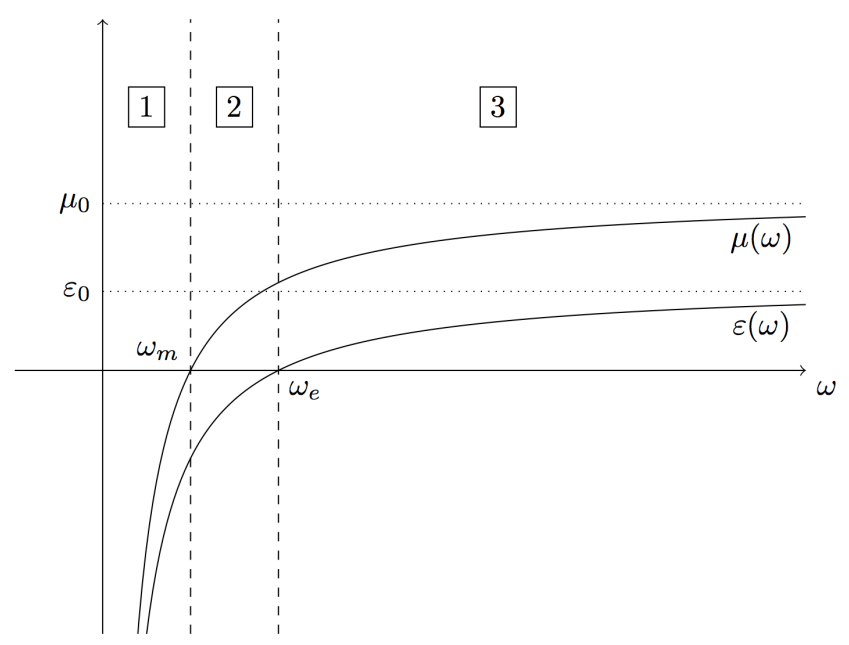

FiguRE 6. Illustration of the three frequency bands for the Drude model for the case $\omega_{e}>\omega_{m}$. Area 1: negative index - area 2: band gap - area 3: classical behaviour.

Notice that although the Drude model is isotropic, it gives rise to backward waves contrarily to non-dispersive models where backward waves can only appear with anisotropy.

\subsubsection{Going back in time}

To obtain a first-order system in the time domain, one injects (27) in (26) and introduces three auxiliary fields $J_{x}, J_{y}$ and $K$ (called the induced electric and magnetic currents) which are the primitives in time of the fields $E_{x}, E_{y}$ and $H: \partial_{t} J_{x}=E_{x}, \partial_{t} J_{y}=E_{y}$ and $\partial_{t} K=H$. We obtain the system for the Drude model in the time domain

$$
\left\{\begin{array}{ccc}
\partial_{t} E_{x}+\omega_{e}^{2} J_{x} & = & \varepsilon_{0}^{-1} \partial_{y} H \\
\partial_{t} J_{x}-E_{x} & = & 0 \\
\partial_{t} E_{y}+\omega_{e}^{2} J_{y} & = & -\varepsilon_{0}^{-1} \partial_{x} H \\
\partial_{t} J_{y}-E_{y} & = & 0 \\
\partial_{t} H+\omega_{m}^{2} K & = & \mu_{0}^{-1}\left(\partial_{y} E_{x}-\partial_{x} E_{y}\right) \\
\partial_{t} K-H & = & 0
\end{array}\right.
$$

The well-posedness of (30) is just a direct application of the classical theories for linear evolution equations like the Hille-Yosida's theory. Moreover, the 6 modes of (30) are given by $\pm \omega_{e}$ and the 4 modes of the Proposition 2.2. In particular, they are all real, so the system (30) is at least weakly stable in the sense of (22). Moreover, we can easily establish the following energy identity

$$
\frac{\mathrm{d}}{\mathrm{d} t}\left(\mathcal{E}_{e}+\mathcal{E}_{m}\right)=0
$$

where

$$
\left\{\begin{aligned}
\mathcal{E}_{e} & =\frac{1}{2} \int_{\mathbb{R}^{2}} \varepsilon_{0}\left(\left|E_{x}\right|^{2}+\left|E_{y}\right|^{2}\right)+\frac{1}{2} \int_{\mathbb{R}_{2}} \varepsilon_{0} \omega_{e}^{2}\left(\left|J_{x}\right|^{2}+\left|J_{y}\right|^{2}\right) \\
\mathcal{E}_{m} & =\frac{1}{2} \int_{\mathbb{R}^{2}} \mu_{0}|H|^{2}+\frac{1}{2} \int_{\mathbb{R}^{2}} \mu_{0} \omega_{m}^{2}|K|^{2} .
\end{aligned}\right.
$$

This implies an estimate of type (19) with $s=0$, i.e. the system (30) is strongly stable, the solution remains bounded for all $t \geq 0$. 


\subsubsection{Classical PMLs for the Drude model}

To construct the classical PMLs for the Drude model, we follow several steps detailed in [5] (see also 1.1.1):

(1) In the frequency domain, we split the field $H$ into $H=H^{x}+H^{y}$ in order to get only one derivative per equation, i.e. the third equation of (26) is replaced by the two equations

$$
\left\{\begin{array}{l}
i \omega \mu(\omega) \widehat{H^{x}}=\partial_{y} \widehat{E_{x}} \\
i \omega \mu(\omega) \widehat{H^{y}}=-\partial_{x} \widehat{E_{y}}
\end{array}\right.
$$

Notice that splitting the electric field is not needed.

(2) We inject (27) in the new split system and use the change of variable (11) with (12);

(3) We introduce the first primitives in times $J_{x}, J_{y}, K^{x}$ and $K^{y}$ as previously and also the second primitives in time $P_{x}, P_{y}, M^{x}$ and $M^{y}$ of $E_{x}, E_{y}, H^{x}$ and $H^{y}$ (notice that subscripts $x$ or $y$ stand for each components of a vector fields like $\mathbf{E}=\left(E_{x}, E_{y}\right)$ but superscripts stand for artificial split scalar fields like $\left.H=H^{x}+H^{y}\right)$.

(4) We go back in time and get the classical Perfectly Matched Layers system for the Drude model

$$
\left\{\begin{array}{ccc}
\partial_{t} E_{x}+\sigma_{y} E_{x}+\omega_{e}^{2} J_{x}+\sigma_{y} \omega_{e}^{2} P_{x} & = & \varepsilon_{0}^{-1} \partial_{y}\left(H^{x}+H^{y}\right), \\
\partial_{t} J_{x}-E_{x} & = & 0, \\
\partial_{t} P_{x}-J_{x} & = & 0, \\
\partial_{t} E_{y}+\sigma_{x} E_{y}+\omega_{e}^{2} J_{y}+\sigma_{x} \omega_{e}^{2} P_{y} & = & -\varepsilon_{0}^{-1} \partial_{x}\left(H^{x}+H^{y}\right), \\
\partial_{t} J_{y}-E_{y} & = & 0, \\
\partial_{t} P_{y}-J_{y} & = & 0, \\
\partial_{t} H^{x}+\sigma_{y} H^{x}+\omega_{e}^{2} K^{x}+\sigma_{y} \omega_{m}^{2} M^{x} & = & \mu_{0}^{-1} \partial_{y} E_{x}, \\
\partial_{t} K^{x}-H^{x} & = & 0, \\
\partial_{t} M^{x}-K^{x} & = & 0, \\
\partial_{t} H^{y}+\sigma_{x} H^{y}+\omega_{m}^{2} K^{y}+\sigma_{x} \omega_{m}^{2} M^{y} & = & -\mu_{0}^{-1} \partial_{x} E_{y}, \\
\partial_{t} K^{y}-H^{y} & = & 0, \\
\partial_{t} M^{y}-K^{y} & = & 0 .
\end{array}\right.
$$

\subsubsection{Numerical simulations}

We now perform a numerical simulation which illustrates the inherent instability of (34). We use the Finite Difference Time Domain (FDTD) method based on the Yee's scheme. For more details about the numerical scheme, see [5]. The computational domain is a square $\Omega=(-20,20)^{2}$. The speed of light is normalized to 1 : $\varepsilon_{0}=\mu_{0}=1$ so $c=1 / \sqrt{\varepsilon_{0} \mu_{0}}=1$ and we take $\omega_{e}=\omega_{m}=2$. All the fields are zeros at the initial time $t=0$ and we add a source term which is a product of a Gaussian function in space centred at $(0,0)$ and a Gaussian pulse in time in order to excite a large spectrum of frequencies: both forward and backward waves are present. The width $L$ of the PMLs is $L=3$ so that the total computational domain is $\Omega=(-20,20)^{2}$ but the physical domain is $(-17,17)^{2}$. In order to reduce numerical reflections at the interface between the physical domain and the PML, the damping terms $\sigma_{x}$ and $\sigma_{y}$ are taken quadratically increasing. At the end of the PMLs, we use the perfect conductor condition : $\left(E_{x}, E_{y}\right)^{T} \times \mathbf{n}=0$.

The Figure 7 shows snapshots of the field $H$ at different times. We clearly observe instabilities. Those do not come from the discretization, the continuous model (34) is inherently unstable: one can refine the mesh and/or reduce the CFL number and get the same phenomenon. Notice that the backward waves are responsible for the instabilities. Indeed the forward waves, the faster ones, are well absorbed by the PML but as soon as the backward waves reach the PML, the solution grows exponentially in time. The Figure 8 illustrates these statements by plotting the logarithm of the energy $\mathcal{E}_{e}+\mathcal{E}_{m}$ defined by (32).

Remark 2.3. We emphasise that the instabilities do not come from the boundaries at the end of the PMLs. Indeed, we will show in Section 3 that the system (34) is inherently unstable even in the free space. Moreover, 

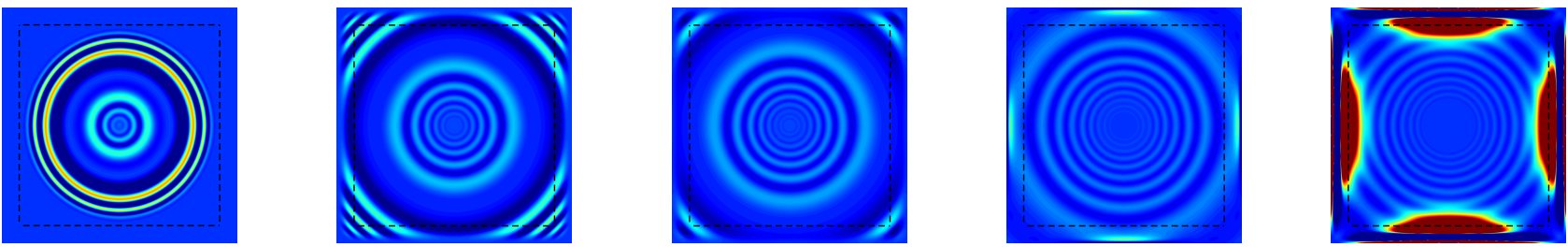

Figure 7. Snapshots of the field $H=H^{x}+H^{y}$ at different times $t$ : the forward waves are well absorbed but the backward waves blow up exponentially

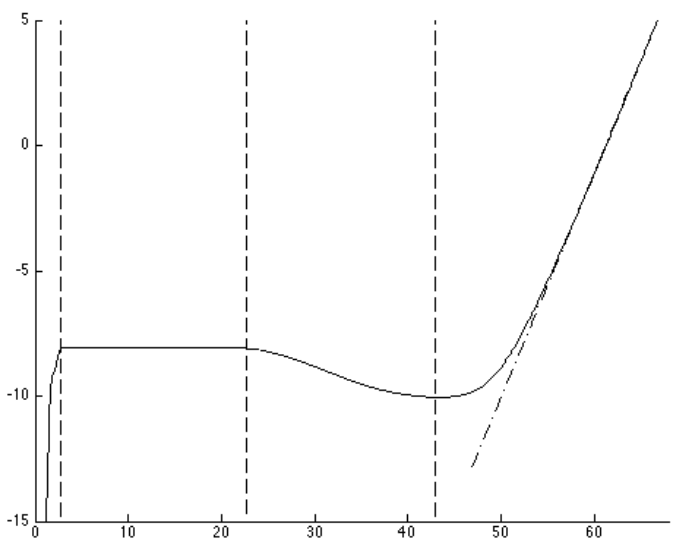

Figure 8. Logarithm of the energy $\mathcal{E}_{e}+\mathcal{E}_{m}$ defined by (32). From $t=0$ to $t \simeq 3$ the energy grows because of the source term. Then the energy is preserved until $t \simeq 22$ when the forward waves, the fastest ones, reach the PML where they are absorbed, so the energy starts decaying. But after $t \simeq 40$, the backward waves reach the PML and the energy starts to grow up exponentially in time, i.e. linearly in a logarithmic scale : the dotted line is a fitting $y=0.9 t-55$.

one can make simulations with larger PMLs so that the outer boundary is far from the interface between the PML and the physical medium,or even in a full PML medium, and observe instabilities before any wave reaches the outer boundary.

\subsection{The case of plasmas: the uniaxial anisotropic plasma model.}

\subsubsection{The model}

A widely used model for describing the physics of cold plasmas is the so-called cold uniaxial anisotropic plasma model given by [21]

$$
\nabla \times \nabla \times \mathbf{E}-\frac{\omega^{2}}{c^{2}} \frac{\underline{\varepsilon}}{\frac{\varepsilon}{(}(\omega)} \mathbf{E}=0
$$

where $\underline{\underline{\varepsilon}}$ is the dielectric tensor. We will restrain ourselves to the $2 \mathrm{D}$ case $^{2}: \mathbf{E}=\left(E_{x}, E_{y}\right)$. For a plasma along the $\mathbf{e}_{y}$ direction, this tensor is given by

$$
\frac{\underline{\underline{\varepsilon}}(\omega)}{\varepsilon_{0}}=\left[\begin{array}{cc}
\varepsilon_{\perp} & 0 \\
0 & \varepsilon_{\|}
\end{array}\right] \quad \text { with } \quad \varepsilon_{\perp}=1, \quad \varepsilon_{\|}=1-\frac{\omega_{p}^{2}}{\omega^{2}}
$$

\footnotetext{
${ }^{2}$ The $3 \mathrm{D}$ case is much harder and is the subject of an ongoing work, whereas the extension of our work about NIMs in $3 \mathrm{D}$ is more or less trivial.
} 
where $\omega_{p}>0$ is the plasma frequency.

Whereas the Drude model is isotropic, the plasma shows different behaviour depending on the values of $\omega$ but also depending on the direction. Notice that the curl operator "switches" the direction so we have the following behaviours (see also equation (37)):

- in the $y$ direction, since $\varepsilon_{\perp}=1$, the medium behaves as a standard dielectric;

- in the $x$ direction, for $\omega>\omega_{p}$ one obtains $\varepsilon_{\|}>0$, so the medium behaves as a dielectric medium, but for $\omega<\omega_{p}$ one obtains $\varepsilon_{\|}<0$ : there is no wave propagation.

\subsubsection{Plane waves analysis}

Computing the dispersion relation of (35) combined with (36) shows that, for a given wave vector $\mathbf{k}=\left(k_{x}, k_{y}\right)$, $\omega$ has to be a solution of

$$
c^{2} k_{x}^{2}+\left(1-\frac{\omega_{p}^{2}}{\omega^{2}}\right) c^{2} k_{y}^{2}=\omega^{2}-\omega_{p}^{2} .
$$

We would like to emphasis that, unlike the Drude model, this one is anisotropic: the modes in (37) depend on $\mathbf{k}$ and not just on $|\mathbf{k}|$.

Proposition 2.4. For all wave vectors $\mathbf{k}=\left(k_{x}, k_{y}\right) \in \mathbb{R}^{2}$, the equation (37) has four real solutions $\pm \omega_{j}(\mathbf{k})$, $j=1,2$ given by

$$
\left\{\begin{array}{l}
\omega_{1}(\mathbf{k})=\frac{\sqrt{2}}{2} \sqrt{c^{2}|\mathbf{k}|^{2}+\omega_{p}^{2}+\sqrt{\Delta_{\mathbf{k}}}}, \\
\omega_{2}(\mathbf{k})=\frac{\sqrt{2}}{2} \sqrt{c^{2}|\mathbf{k}|^{2}+\omega_{p}^{2}-\sqrt{\Delta_{\mathbf{k}}}},
\end{array}\right.
$$

where $\Delta_{\mathbf{k}}=\left(\left(\omega_{p}^{2}+c k_{y}\right)^{2}+c^{2} k_{x}^{2}\right)\left(\left(\omega_{p}^{2}-c k_{y}\right)^{2}+c^{2} k_{x}^{2}\right)$. Moreover, one can show that the modes $\pm \omega_{1}(\mathbf{k})$ are forward in all the directions whereas the modes $\pm \omega_{2}(\mathbf{k})$ are forward in the $\mathbf{e}_{y}$ direction but backward in the $\mathbf{e}_{x}$ direction.

\subsubsection{Time-domain system and classical PMLs}

Following the same idea of Section 2.1.3, the first-order system corresponding to (35) with (36) is

$$
\left\{\begin{array}{ccc}
\partial_{t} E_{x} & = & c^{2} \partial_{y} H \\
\partial_{t} E_{y}+\omega_{p}^{2} \phi_{p} & = & -c^{2} \partial_{x} H \\
\partial_{t} \phi_{p}-E_{y} & = & 0 \\
\partial_{t} H & = & \partial_{y} E_{x}-\partial_{x} E_{y}
\end{array}\right.
$$

and, as in Section 2.1.4, the classical PMLs applied to (39) give

$$
\left\{\begin{array}{ccc}
\partial_{t} E_{x}+\sigma_{y} E_{x} & = & c^{2} \partial_{y}\left(H^{x}+H^{y}\right) \\
\partial_{t} E_{y}+\sigma_{x} E_{y}+\omega_{p}^{2} \phi_{p}+\sigma_{x} \omega_{p}^{2} \Phi_{p} & = & -c^{2} \partial_{x}\left(H^{x}+H^{y}\right) \\
\partial_{t} \phi_{p}-E_{y} & = & 0 \\
\partial_{t} \Phi_{p}-\phi_{p} & = & 0 \\
\partial_{t} H^{x}+\sigma_{y} H^{x} & = & \partial_{y} E_{x} \\
\partial_{t} H^{y}+\sigma_{x} H^{y} & = & -\partial_{x} E_{y} .
\end{array}\right.
$$

\subsubsection{Numerical simulations}

We perform a numerical experiment of (40) following the same procedure of the Section 2.1.5. The parameters are $\omega_{p}=4, \Omega=(-10,10) \times(-20,20)$ and the source is now harmonic in time, i.e. of the form $\sin \left(\omega_{0} t\right)$ in order to really emphasise the phenomenon. We take here $\omega_{0}=2$ which leads to $\varepsilon_{\|}=-3$. The PMLs are still of width $L=3$. The Figure 9 shows some snapshots of the field $H=H^{x}+H^{y}$ at different times. We observe that the PML in the $y$ direction does not give rise to instabilities whereas the PML in the $x$ direction appears to be unstable. 

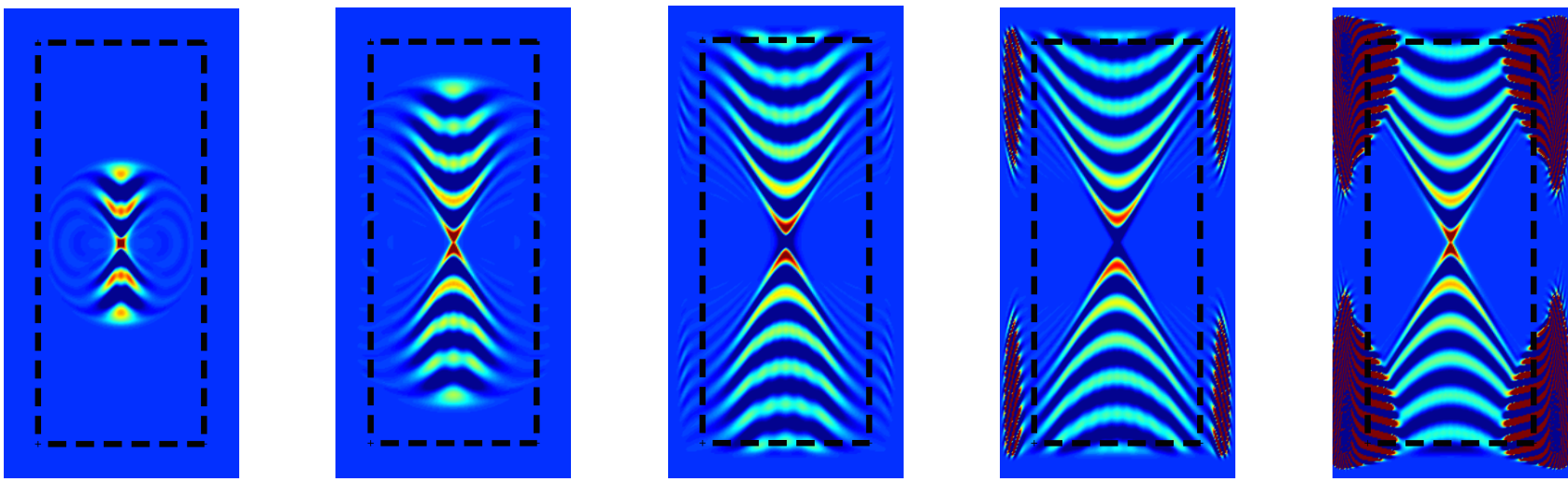

Figure 9. Snapshots of the field $H=H^{x}+H^{y}$ at different times $t$.

\section{Designing Stable PMLs for Negative indeX Metamaterials and Plasmas}

This section explains how the stability criterion (23) in Theorem 1.3 can be extended to dispersive models and more general changes of variable. This allows us to understand the instabilities observed numerically and helps us to design new stable PMLs.

The dispersive nature of the considered systems adds some difficulties compared to the previous case of Section 1.2.2. First, the original dispersion relation is no more homogeneous, so taking large frequencies $|\mathbf{k}|$ does not amount taking small values of $\sigma$ any more. Thus we need a new definition of uniform stability (Definition 3.1). Second, the number of added modes after the change of variable (43) is unknown (previously we knew that it would add $m$ modes). Third, the geometrical criterion of stability for non dispersive systems cannot be trivially extended to dispersive systems since the shapes of the slowness curves now depend on the frequency.

\subsection{A generalized necessary criterion of stability for a class of dispersive systems}

The goal of this section is to describe how the analysis of the Section 1.2.2 can be extended to dispersive models but also to more general changes of variable than (12) in order to design new stable PMLs. We present here the main ideas, all the details can be found in [5].

We consider a class of dispersive systems described by 2 D systems of unknown $\mathbf{u} \in \mathbb{R}^{m}$ of the form

$$
\partial_{t} \mathbf{u}+A_{x} \partial_{x} \mathbf{u}+A_{y} \partial_{y} \mathbf{u}+B \mathbf{u}=0
$$

where $A_{x}, A_{y}$ and $B$ are real constant $m \times m$ matrices. The previous case in Section 1.2 .2 corresponds to $B=0$. Notice that both the Drude model and the 2D uniaxial plasma model are particular cases of this kind of system. The dispersion relation of (41) writes

$$
F\left(\omega, k_{x}, k_{y}\right)=0 \quad \text { where } \quad F\left(\omega, k_{x}, k_{y}\right)=\operatorname{det}\left(i \omega I_{m}-i k_{x} A_{x}-i k_{y} A_{y}+B\right) .
$$

Again, this is a polynomial equation in $\omega$ of order $m$ which gives $m$ modes $\omega_{j}(\mathbf{k}), j=1, \ldots, m$ assumed to be real.

In order to design new stable PMLs, we shall allow more general changes of variable. Instead of (12), we look for

$$
\alpha(x)=\left(1+\frac{\sigma}{i \omega \psi(\omega)}\right)^{-1}
$$

where $\psi(\omega)$ is a real valued function of $\omega$, and $\sigma>0$ the damping term supposed to be constant. In practice, $\psi$ will be a rational function in $\omega$ in order to ensure that one obtains local differential operators after going back 
in time. The classical PMLs corresponds to $\psi(\omega)=1$. The change of variable (11) combined with (43) modifies the dispersion relation (42) into

$$
F\left(\omega,\left(1+\frac{\sigma}{i \omega \psi(\omega)}\right)^{-1} k_{x}, k_{y}\right)=0
$$

which gives $M \geq m$ modes $\omega_{j}(\mathbf{k}, \sigma)$.

Since $F$ is no longer homogeneous (compared to Section 1.2.2), looking for large frequencies $|\mathbf{k}|$ does not amount looking for small values of $\sigma$ any more. This motivates the introduction of the definition of uniform stability with respect to the parameter $\sigma$.

Definition 3.1. A family of systems corresponding to (44) parametrized by $\sigma \geq 0$ is said to be uniformly stable in $\sigma$ if

$$
\forall \sigma \geq 0, \forall \mathbf{k} \in \mathbb{R}^{2}, \forall j \in\{1, \ldots, M\}, \quad \operatorname{Im} \omega_{j}(\mathbf{k}, \sigma) \geq 0
$$

In [5], the following result is proven:

Theorem 3.2. For the dispersive system (41), a necessary condition of uniform stability for the family of PML systems corresponding to (44) is that all the modes $\omega(\mathbf{k})$ solving the original dispersion relation (42) verify

$$
\forall \mathbf{k} \in \mathbb{R}^{2}, \quad \frac{\left(\mathbf{v}_{\mathbf{p}}(\omega(\mathbf{k})) \cdot \mathbf{e}_{x}\right)\left(\mathbf{v}_{\mathbf{g}}(\omega(\mathbf{k})) \cdot \mathbf{e}_{x}\right)}{\psi(\omega(\mathbf{k}))} \geq 0
$$

This allows us to understand why the classical PMLs are unstable for both the Drude model and the uniaxial plasma model. Indeed, in this case $\psi(\omega)=1$ and the necessary condition means that all the modes in the $x$ direction must be forward, as in Theorem 1.3. But Propositions 2.2 and 2.4 state that there are always a backward mode in the $x$ direction for both models, so the corresponding systems are unstable. This explains the instabilities observed numerically in Section 2.1.5 and 2.2.4.

\subsection{New stable PMLs for the Drude model}

The Theorem 3.2 provides us a simple way to design new PMLs for the Drude model that we can expect to be stable, by choosing carefully the function $\psi$. First notice that $\omega_{1}(\mathbf{k}) \geq \max \left(\omega_{e}, \omega_{m}\right)$ and $\omega_{2}(\mathbf{k}) \leq \min \left(\omega_{e}, \omega_{m}\right)$ so, for a given frequency $\omega$, only one of the three following situations can occur:

- for $\omega>\max \left(\omega_{e}, \omega_{m}\right)$, the waves propagate forward: one must choose $\psi$ such that $\psi(\omega)>0$;

- for $\omega<\min \left(\omega_{e}, \omega_{m}\right)$, the waves propagate backward: one must impose that $\psi(\omega)<0$;

- in the band gap $\left(\min \left(\omega_{e}, \omega_{m}\right), \max \left(\omega_{e}, \omega_{m}\right)\right), \omega$ is not in the range of the modes $\omega_{j}(\mathbf{k})$, so the sign of $\psi$ does not matter.

Of course, the choice of $\psi$ is not unique. However, $\psi$ should be chosen in order to keep the system as simple as possible after going back in time. A pertinent choice (whose premises already appeared in the physical literature $[9,11,14,19])$ is

$$
\psi(\omega)=\left(1-\frac{\omega_{*}^{2}}{\omega^{2}}\right)
$$

where $\omega_{*} \in\left[\min \left(\omega_{e}, \omega_{m}\right), \max \left(\omega_{e}, \omega_{m}\right)\right]$. Notice that for $\omega_{*}=\omega_{e}\left(\operatorname{resp} . \omega_{*}=\omega_{m}\right)$ one gets $\psi(\omega)=\varepsilon(\omega) / \varepsilon_{0}$ (resp. $\left.\psi(\omega)=\mu(\omega) / \mu_{0}\right)$. 
The computations to go back in time after the change of variable using (43) with (47) are quite heavy but straightforward. We obtain (see [5] for the details) the new PML system for the Drude model:

$$
\left\{\begin{array}{ccc}
\partial_{t} E_{x}+\omega_{e}^{2} J_{x}+\varepsilon_{0}^{-1} \sigma_{y}\left(E_{x}+\widetilde{E}_{x}\right) & = & \varepsilon_{0}^{-1} \partial_{y}\left(H^{x}+H^{y}\right), \\
\partial_{t} J_{x}-E_{x} & = & 0, \\
\partial_{t} \widetilde{E}_{x}+\omega_{*}^{2} \widetilde{J}_{x}+\left(\omega_{*}^{2}-\omega_{e}^{2}\right) J_{x} & = & 0, \\
\partial_{t} \widetilde{J}_{x}-\widetilde{E}_{x} & = & 0, \\
\partial_{t} E_{y}+\omega_{e}^{2} J_{y}+\varepsilon_{0}^{-1} \sigma_{x}\left(E_{y}+\widetilde{E}_{y}\right) & = & -\varepsilon_{0}^{-1} \partial_{x}\left(H^{x}+H^{y}\right), \\
\partial_{t} J_{y}-E_{y} & = & 0, \\
\partial_{t} \widetilde{E}_{y}+\omega_{*}^{2} \widetilde{J}_{y}+\left(\omega_{e}^{2}-\omega_{*}^{2}\right) J_{y} & = & 0, \\
\partial_{t} \widetilde{J}_{y}-\widetilde{E}_{y} & = & 0, \\
\partial_{t} H^{x}+\omega_{m}^{2} K^{x}+\mu_{0}^{-1} \sigma_{y}\left(H^{x}+\widetilde{H}^{x}\right) & = & \mu_{0}^{-1} \partial_{y} E_{x}, \\
\partial_{t} K^{x}-H^{x} & = & 0, \\
\partial_{t} \widetilde{H}^{x}+\omega_{*}^{2} \widetilde{K}^{x}+\left(\omega_{*}^{2}-\omega_{m}^{2}\right) K^{x} & = & 0, \\
\partial_{t} \widetilde{K}^{x}-\widetilde{K}^{x} & = & 0, \\
\partial_{t} H^{y}+\omega_{m}^{2} K^{y}+\mu_{0}^{-1} \sigma_{y}\left(H^{y}+\widetilde{H}^{y}\right) & = & -\mu_{0}^{-1} \partial_{x} E_{y}, \\
\partial_{t} K^{y}-H^{y} & = & 0, \\
\partial_{t} \widetilde{H}^{y}+\omega_{*}^{2} \widetilde{K}^{y}+\left(\omega_{m}^{2}-\omega_{*}^{2}\right) K^{y} & = & 0, \\
\partial_{t} \widetilde{K}^{y}-\widetilde{K}^{y} & = & 0 .
\end{array}\right.
$$

In the general case, the system (48) has 16 unknowns. The choice $\omega_{*}=\omega_{e}$ (resp. $\omega_{*}=\omega_{m}$ ) which corresponds to $\psi(\omega)=\varepsilon(\omega) / \varepsilon_{0}$ (resp. $\left.\psi(\omega)=\mu(\omega) / \mu_{0}\right)$ simplifies it. Indeed, we have $\omega_{e}^{2}-\omega_{*}^{2}=0$ (resp. $\left.\omega_{m}^{2}-\omega_{*}^{2}=0\right)$ so the fields $\widetilde{E}_{x}, \widetilde{E}_{y}, \widetilde{J}_{x}$ and $\widetilde{J}_{y}$ (resp. $\widetilde{H}^{x}, \widetilde{H}^{y}, \widetilde{K}^{x}$ and $\widetilde{K}^{y}$ ) are no longer needed and the system will have only 12 unknowns instead of 16 . Moreover, if $\omega_{e}=\omega_{m}$, both of the two simplification appear and the system will have only 8 unknowns.

As expected, the choice (47) leads to a stable system (see [5] for a proof):

Proposition 3.3. Assume $\sigma_{x}, \sigma_{y} \geq 0$ and $\omega_{*} \in\left[\min \left(\omega_{e}, \omega_{m}\right), \max \left(\omega_{e}, \omega_{m}\right)\right]$. Then the family of systems (48) parametrised by $\sigma_{x}$ and sigma is $_{y}$ uniformly stable.

We now perform numerical simulations to illustrate the stability of the system (48). To begin with, we reproduce exactly the same experiment as in Section 2.1.5 with the same parameters, the same source, etc. but of course with the new stable PMLs (48) instead of the classical ones (34). We choose here $\omega_{*}=\omega_{e}$ in order to reduce the number of unknowns. As expected, the system remains stable during all the computation with a final time of $T=250$. The Figure 10 shows snapshots of the field $H$ at different times. We clearly see that the system remains stable. Both of forward and backward waves are well absorbed.
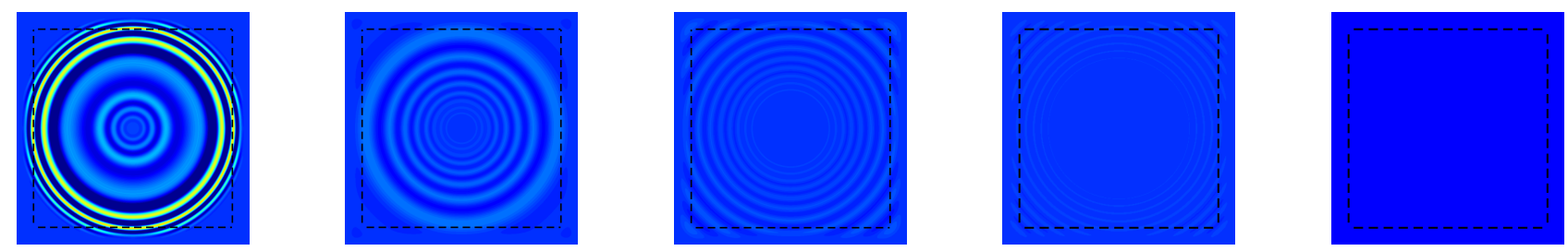

Figure 10. Snapshots of the field $H=H^{x}+H^{y}$ at different times $t$. Both forward and backward waves are well absorbed.

The new stable PMLs can be used to illustrate the negative index behaviour of the Drude model. We perform a simulation of a transmission problem between the vacuum and a Drude medium surrounded by PMLs and new stable PMLs (see Figure 11) with a spacial source localized in the vacuum. We used $\omega_{e}=\omega_{m}=2$ but here 
we take a periodic time source $h(t)=\sin \left(\omega_{0} t\right)$ where the frequency $\omega_{0}$ is taken equal to $\omega_{e} / \sqrt{2}=\omega_{m} / \sqrt{2}$. With this choice, we have $\varepsilon\left(\omega_{0}\right)=\mu\left(\omega_{0}\right)=-1$ so the effective index of the metamaterial is -1 (see Remark 2.1).

The Snell law applied to our problem gives us the change of direction of the waves due to the change of medium. It remains valid even for negative index and leads to the so-called negative refraction [22]. Thus, we can predict a symmetric refocalisation of the wave in the metamaterial, as shown in Figure 11. The Figure 12 shows snapshots of the field $H$ for this experiment. Notice that the solution remains stable and that $H$ actually refocuses in the Drude medium.

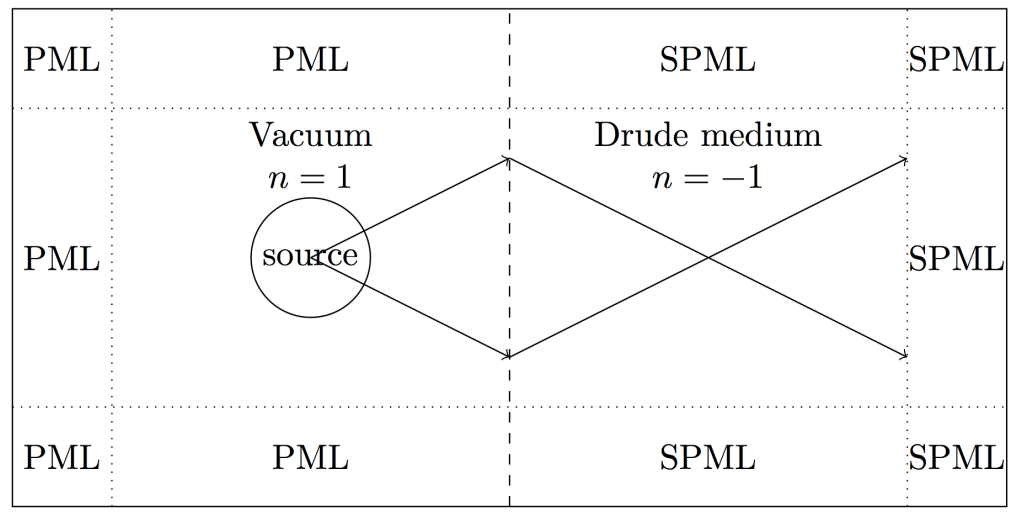

Figure 11. The geometry of the transmission problem and the effect of the negative index according to the Snell law (SPML = stable PML).
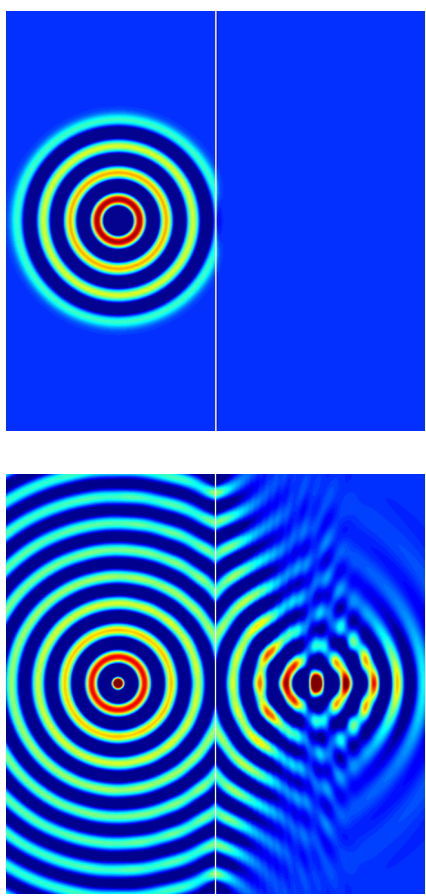
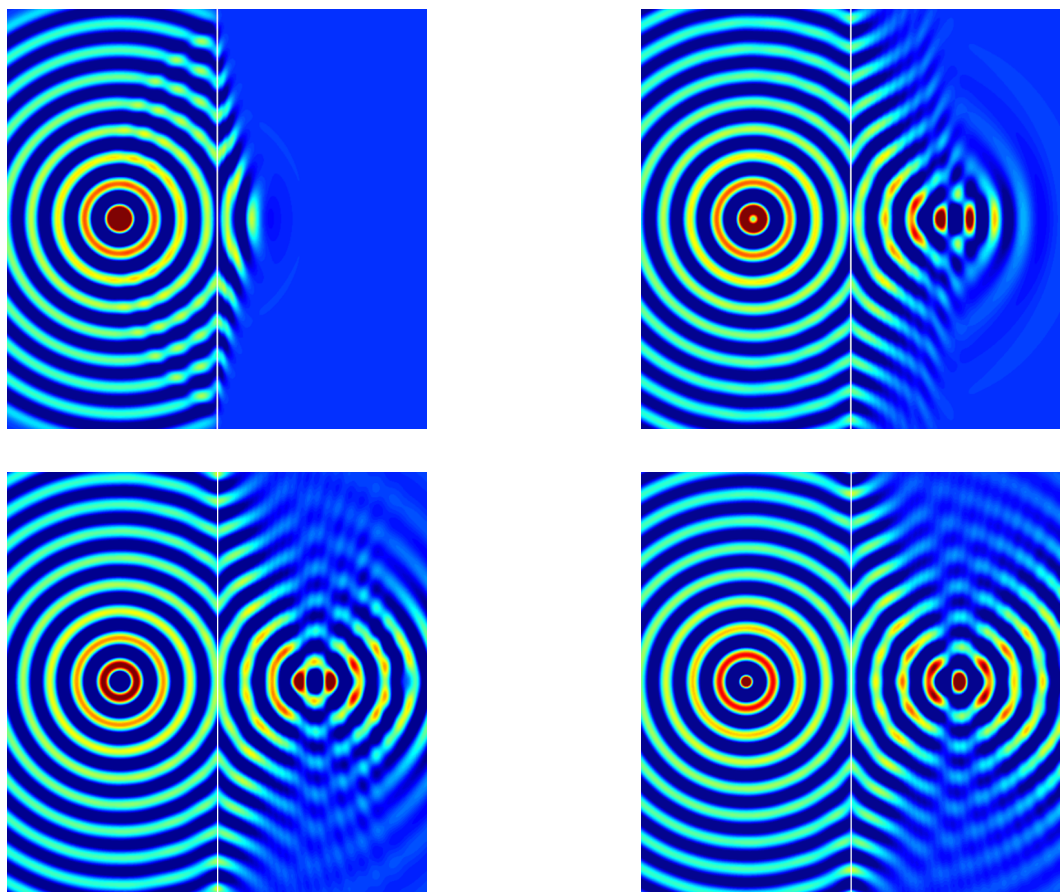

Figure 12. Refocalisation of the waves due to the negative index of the Drude model (the PMLs are not represented). 


\subsection{Application to the uniaxial anisotropic plasma model}

We can use again the idea of the Section 3.2, but now the medium is anisotropic so we must treat the two directions separately:

- in the $y$ direction, both modes $\omega_{1}(\mathbf{k})$ and $\omega_{2}(\mathbf{k})$ are forward: the classical PMLs should work, thus we take $\psi(\omega)=1$.

- for the $x$ direction, notice that $\omega_{1}(\mathbf{k}) \geq \omega_{p}$ and $\omega_{2}(\mathbf{k}) \leq \omega_{p}$. Thus, for a given frequency $\omega$, only one of the two following situations can occur: for $\omega>\omega_{p}$ the waves propagate forward, so we must choose $\psi$ such that $\psi(\omega)>0$, and for $\omega<\omega_{p}$ the waves propagate backward, so we must choose $\psi$ such that $\psi(\omega)<0$.

The originality here is that the PMLs will be different depending on the direction. We take

$$
\psi_{x}(\omega)=1-\frac{\omega_{p}^{2}}{\omega^{2}}
$$

for the $x$ direction and we use the classical PMLs $\psi_{y}(\omega)=1$ in the $y$ direction.

Again, we can derive a time-domain system corresponding to this choice:

$$
\left\{\begin{array}{ccc}
\partial_{t} E_{x}+\sigma_{y} E_{x} & = & c^{2} \partial_{y}\left(H^{x}+H^{y}\right), \\
\partial_{t} E_{y}+\sigma_{x} E_{y}+\omega_{p}^{2} \phi_{p} & = & -c^{2} \partial_{x}\left(H^{x}+H^{y}\right), \\
\partial_{t} \phi_{p}-E_{y} & = & 0 \\
\partial_{t} H^{x}+\sigma_{y} H^{x} & = & \partial_{y} E_{x} \\
\partial_{t} H^{y}+\sigma_{x} K^{y} & = & -\partial_{x} E_{y}, \\
\partial_{t} K^{y}-\omega_{p}^{2} M^{y}-H^{y} & = & 0, \\
\partial_{t} M^{y}-K^{y} & = & 0 .
\end{array}\right.
$$

As in the Drude model, the choice of $\psi_{x}$ and $\psi_{y}$ leads to stable PMLs:

Proposition 3.4. Assume $\sigma_{x}, \sigma_{y} \geq 0$. Then the family of systems (50) parametrised by $\sigma_{x}$ and sigma $a_{y}$ is uniformly stable.

We now perform a numerical simulation to illustrate the above proposition. We take the same parameters of the Section 2.2.4 and use the new PML system (50). As expected, the solution remains stable during all the computation time as shown in Figure 13.

Remark 3.5. Instead of (50), in the simulation we employ a different (non-split) PML formulation. A split formulation is presented here for consistency with the rest of the article. The two systems are completely equivalent.

\section{Conclusion}

In the first part, we did a brief survey on classical PMLs in non-dispersive media: construction, necessary criterion of stability and application to some wave models. In the second part, we extended these results to a large class of dispersive media and to more general PMLs than the classical ones. A new necessary criterion of stability has been established. Thanks to it, we proposed a method to design new PMLs that takes into account the backward waves. We applied these ideas to the Drude model (negative index metamaterial) and the uniaxial anistropic plasma model. For both models, numerical simulations have been performed to illustrate the inherent instabilities of classical PMLs and the stability of the new ones.

Here we merely present the results, all the details and proofs can be found in a forthcoming paper [5]. We would like to remark that our approach does not apply when both forward and backward waves occur at the same frequency, which is the case for some anisotropic media such as elastodynamics [3] or for more complicated backward-wave structures [17]. It also happens for the 3D case in plasmas, which is the subject of an ongoing work. 

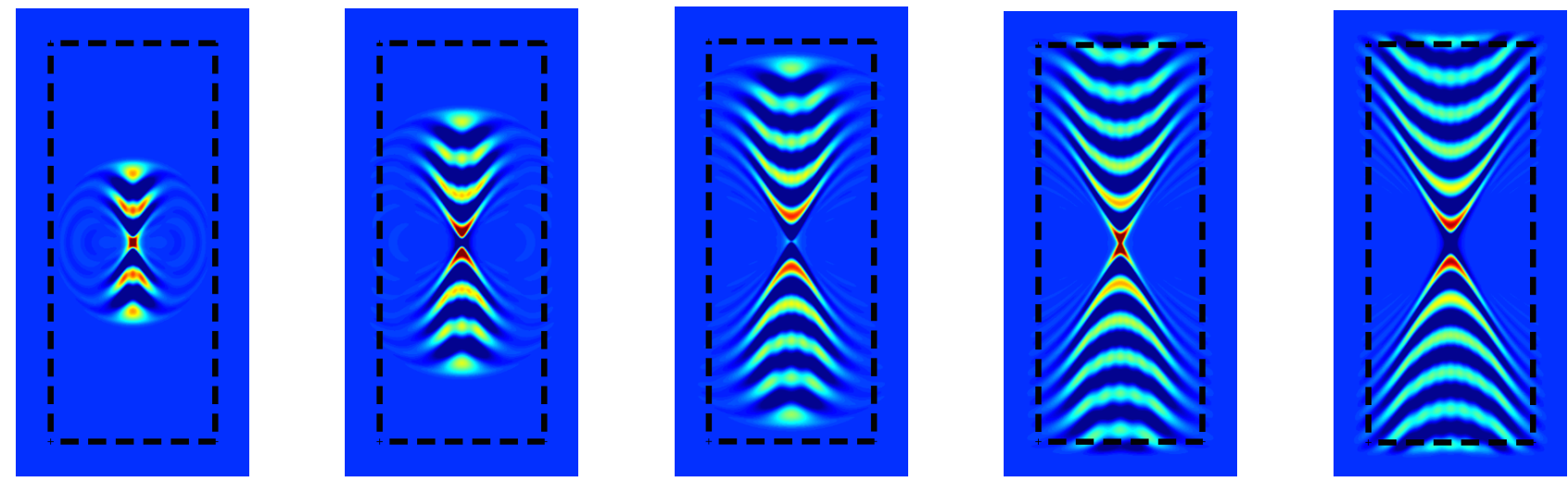

Figure 13. Snapshots of the field $H=H^{x}+H^{y}$ at different times $t$.

Acknowledgement: the authors thank Guillaume Chicaud for his work on cold plasmas during his internship at the POems team.

\section{REFERENCES}

[1] Saul Abarbanel and David Gottlieb. A mathematical analysis of the PML method. Journal of Computational Physics, 134(2):357-363, 1997.

[2] Daniel Appelö, Thomas Hagstrom, and Gunilla Kreiss. Perfectly matched layers for hyperbolic systems: general formulation, well-posedness, and stability. SIAM Journal on Applied Mathematics, 67(1):1-23, 2006.

[3] Eliane Bécache, Sandrine Fauqueux, and Patrick Joly. Stability of perfectly matched layers, group velocities and anisotropic waves. Journal of Computational Physics, 188(2):399-433, 2003.

[4] Eliane Bécache and Patrick Joly. On the analysis of Bérenger's perfectly matched layers for maxwell's equations. ESAIM: Mathematical Modelling and Numerical Analysis, 36(01):87-119, 2002.

[5] Eliane Bécache, Patrick Joly, and Valentin Vinoles. On the analysis of perfectly matched layers for a class of dispersive media: Application to negative index metamaterials. To be submitted.

[6] Jean-Pierre Bérenger. A perfectly matched layer for the absorption of electromagnetic waves. Journal of computational physics, 114(2):185-200, 1994.

[7] José A Bittencourt. Fundamentals of plasma physics. Springer, 2004.

[8] Weng Cho Chew and William H Weedon. A 3d perfectly matched medium from modified Maxwell's equations with stretched coordinates. Microwave and optical technology letters, 7(13):599-604, 1994.

[9] Davi Correia and Jian-Ming Jin. 3D-FDTD-PML analysis of left-handed metamaterials. Microwave and optical technology letters, 40(3):201-205, 2004.

[10] Tie Jun Cui, David R Smith, and Ruopeng Liu. Metamaterials: theory, design, and applications. Springer, 2010.

[11] Steven A Cummer. Perfectly matched layer behavior in negative refractive index materials. Antennas and Wireless Propagation Letters, IEEE, 3(1):172-175, 2004.

[12] Edouard Demaldent and Sébastien Imperiale. Perfectly matched transmission problem with absorbing layers: Application to anisotropic acoustics in convex polygonal domains. International Journal for Numerical Methods in Engineering, 96(11):689$711,2013$.

[13] Julien Diaz and Patrick Joly. A time domain analysis of pml models in acoustics. Computer methods in applied mechanics and engineering, 195(29):3820-3853, 2006.

[14] XT Dong, XS Rao, YB Gan, B Guo, and WY Yin. Perfectly matched layer-absorbing boundary condition for left-handed materials. Microwave and Wireless Components Letters, IEEE, 14(6):301-303, 2004.

[15] Patrick Joly. An elementary introduction to the construction and the analysis of perfectly matched layers for time domain wave propagation. SeMA Journal, 57(1):5-48, 2012.

[16] Heinz-Otto Kreiss and Jens Lorenz. Initial-boundary value problems and the Navier-Stokes equations, volume 136. Academic press, 1989.

[17] Po-Ru Loh, Ardavan F Oskooi, Mihai Ibanescu, Maksim Skorobogatiy, and Steven G Johnson. Fundamental relation between phase and group velocity, and application to the failure of perfectly matched layers in backward-wave structures. Physical Review E, 79(6):065601, 2009.

[18] John Brian Pendry. Negative refraction makes a perfect lens. Physical review letters, 85(18):3966, 2000. 
[19] Yan Shi, Yun Li, and Chang-Hong Liang. Perfectly matched layer absorbing boundary condition for truncating the boundary of the left-handed medium. Microwave and optical technology letters, 48(1):57-63, 2006.

[20] DR Smith, JB Pendry, and MCK Wiltshire. Metamaterials and negative refractive index. Science, 305(5685):788-792, 2004.

[21] Thomas H Stix. Waves in plasmas. Springer, 1992.

[22] Richard W Ziolkowski and Ehud Heyman. Wave propagation in media having negative permittivity and permeability. Physical review E, 64(5):056625, 2001. 